\title{
Acute Pneumonia and Empyema Caused by Mycobacterium Intracellulare
}

\author{
Sang-Un Park ${ }^{1}$, Won-Jung Koh ${ }^{1}$, O Jung Kwon ${ }^{1}$, Hye Yun Park ${ }^{1}$, Hyun Jung Jun ${ }^{1}$, \\ Eun-Jeong Joo ${ }^{1}$, Nam Yong Lee ${ }^{2}$, Tae Sung Kim ${ }^{3}$, Kyung Soo $\mathrm{Lee}^{3}$ and Young Kil Park ${ }^{4}$
}

\begin{abstract}
Nontuberculous mycobacterial infection is rarely accompanied by pleural involvement. We report a very rare case of M. intracellulare pulmonary disease with pleural empyema. A 56-year-old man was admitted to our hospital because of fever, purulent sputum and pleuritic chest pain. A chest radiograph and CT revealed pneumonic consolidation in the left lower lobe and loculated hydropneumothorax. The sputum smear was positive for acid fast bacilli. The aspirated pleural fluid was grossly purulent and the smear of the pleural effusion was also positive for acid fast bacilli. M. intracellulare was identified by culture and PCR from sputum and pleural fluid specimens. The patient improved with percutaneous tube drainage of the purulent effusion and antibiotic treatment including clarithromycin, rifampicin, ethambutol and streptomycin.
\end{abstract}

Key words: atypical mycobacteria, Mycobacterium avium complex, pleural effusion, pleural empyema

(DOI: 10.2169/internalmedicine.45.1665)

\section{Introduction}

Although the clinical and radiological features of nontuberculous mycobacterial (NTM) infection resemble those of tuberculosis, acute pneumonia or pleural empyema is very rare in cases of NTM pulmonary disease (1). Here, we report a case of acute pneumonia accompanied with pleural empyema caused by $M$. intracellulare.

\section{Case Report}

A 56-year-old man was referred to our hospital for further examination and management of pneumonia. He was a nonsmoker and worked as a farmer. He had a history of previous treatment for pulmonary tuberculosis 30 years earier. He had a chronic cough and mild exertional dyspnea that had not changed over the past several years; he has followed for "bronchial asthma" at a private clinic. He presented with a 4-day history of acute-onset fever, chill, purulent sputum and pleuritic chest pain. He was diagnosed as having community-acquired pneumonia initially, and received intravenous cephalosporin and aminoglycoside. Six days after hospital admission, the patient failed to show clinical improvement. He was then transferred to the emergency room of our hospital.

Physical examination showed that the patient was $160 \mathrm{~cm}$ tall and weighed $53 \mathrm{~kg}$. His pulse rate was 115 beats $/ \mathrm{min}$ and his blood pressure was $104 / 58 \mathrm{mmHg}$ with intravenous infusion of dopamine $10 \mu / \mathrm{kg} / \mathrm{min}$. His body temperature was $37.7^{\circ} \mathrm{C}$ and the respiratory rate was 22 breaths/min. Chest examination revealed coarse crackles over the left hemithorax. There was no lymphadenopathy.

The leukocyte count was $13,050 / \mu \mathrm{L}$, with a differential of $68 \%$ neutrophils. The erythrocyte sedimentation rate was $82 \mathrm{~mm} / \mathrm{h}$ and CRP increased to $23.72 \mathrm{mg} / \mathrm{dL}$ (Table 1). A human immunodeficiency virus antibody test was negative. A chest radiograph and CT revealed pneumonic consolidation in the left lower lobe and loculated hydropneumothorax suggesting a bronchopleural fistula (Fig. 1). Numerous acid-

\footnotetext{
${ }^{1}$ Division of Pulmonary and Critical Care Medicine, Department of Medicine, Samsung Medical Center, Sungkyunkwan University School of Medicine, Seoul, Korea, ${ }^{2}$ Laboratory Medicine, Samsung Medical Center, Sungkyunkwan University School of Medicine, Seoul, Korea, ${ }^{3}$ Department of Radiology, Samsung Medical Center, Sungkyunkwan University School of Medicine, Seoul, Korea and ${ }^{4}$ Korean Institute of Tuberculosis, Korean National Tuberculosis Association, Seoul, Korea

Received for publication November 25, 2005; Accepted for publication June 5, 2006

Correspondence to Dr. Won-Jung Koh, Division of Pulmonary and Critical Care Medicine, Department of Medicine, Samsung Medical Center, Sungkyunkwan University School of Medicine, 50 Irwon-dong, Gangnam-gu, Seoul 135-710, Republic of Korea
} 
Table 1. Laboratory Test Results on Admission

\begin{tabular}{|c|c|c|c|}
\hline \multicolumn{2}{|c|}{ Hematologic laboratory findings } & \multirow{2}{*}{$\begin{array}{l}\text { LDH } \\
\text { BUN }\end{array}$} & \multirow{2}{*}{$\begin{array}{l}622 \mathrm{IU} / \mathrm{L} \\
12.5 \mathrm{mg} / \mathrm{dL}\end{array}$} \\
\hline WBC & $13,050 / \mu \mathrm{L}$ & & \\
\hline $\mathrm{Hb}$ & $14.2 \mathrm{~g} / \mathrm{dL}$ & $\mathrm{Cr}$ & $1.08 \mathrm{mg} / \mathrm{dL}$ \\
\hline Het & $41.3 \%$ & Uric acid & $3.7 \mathrm{mg} / \mathrm{dL}$ \\
\hline Platelet & $547,000 / \mu \mathrm{L}$ & $\mathrm{Na}$ & $135 \mathrm{mEq} / \mathrm{L}$ \\
\hline Differential count & & $\mathrm{K}$ & $3.6 \mathrm{mEq} / \mathrm{L}$ \\
\hline Neutrophils & $68 \%$ & $\mathrm{Cl}$ & $97 \mathrm{mEq} / \mathrm{L}$ \\
\hline Lymphocytes & $17 \%$ & $\mathrm{Ca}$ & $8.4 \mathrm{mg} / \mathrm{dL}$ \\
\hline Monocytes & $12 \%$ & $P$ & $3.8 \mathrm{mg} / \mathrm{dL}$ \\
\hline \multirow[t]{2}{*}{ Eosinophils } & $3 \%$ & $\mathrm{Mg}$ & $1.9 \mathrm{mg} / \mathrm{dL}$ \\
\hline & & CRP & $23.72 \mathrm{mg} / \mathrm{dL}$ \\
\hline \multicolumn{2}{|c|}{ Blood chemical findings } & \multicolumn{2}{|c|}{ Pleural fluid examinations } \\
\hline Total protein & $5.5 \mathrm{~g} / \mathrm{dL}$ & WBC & $46,870 / \mu \mathrm{L}$ \\
\hline Albumin & $2.3 \mathrm{~g} / \mathrm{dL}$ & Differential count & \\
\hline Total bilirubin & $0.6 \mathrm{mg} / \mathrm{dL}$ & Neutrophils & $100 \%$ \\
\hline AST & $17 \mathrm{U} / \mathrm{L}$ & Lymphocytes & $0 \%$ \\
\hline ALT & $15 \mathrm{U} / \mathrm{L}$ & ADA & $146 \mathrm{U} / \mathrm{L}$ \\
\hline
\end{tabular}

fast bacilli (AFB) were seen in multiple sputum specimens (4+). The aspirated pleural fluid was grossly purulent and the fluid contained more than $46,870 \mathrm{WBCs} / \mu \mathrm{L}$ (with a differential of $100 \%$ neutrophils) with a $\mathrm{pH}$ of 7.2. The level of adenosine deaminase in the effusion was markedly elevated (146 U/L) (Table 1). Because of the small amount of aspirated pleural fluid, the biochemical analysis of the effusion could not be performed. Stain for acid-fast bacilli (AFB) of the pleural effusion was also strongly positive $(3+)$. Culture findings for bacteria were subsequently negative.

The patient was then started on a four-drug regimen of isoniazid (300 mg/day), rifampicin (600 mg/day), ethambutol $(1,200 \mathrm{mg} /$ day $)$, and pyrazinamide $(1,500 \mathrm{mg} /$ day $)$ for presumptive diagnosis of pulmonary tuberculosis and tuberculous empyema. Percutaneous tube drainage of pleural fluid was also initiated. After the draining tube was positioned, $50-100 \mathrm{~mL}$ of purulent effusion was drained daily and an air leak persisted for 2 days, indicating a bronchopleural fistula was present. The air leak stopped spontaneously after 2 days. The draining tube was discontinued 12 days after it was placement.

However, nucleic acid amplification tests for M. tuberculosis in sputum and pleural fluid were all negative using a commercial DNA probe (Gen-Probe Amplified Mycobacterium Tuberculosis Direct Test; Gen-Probe Inc, San Diego,
CA, USA). A purified protein derivative skin test was negative. Then, we performed a polymerase chain reaction (PCR) method to identify the causative agent. The result showed a positive result for Mycobacterium intracellulare. Based on the clinical findings and laboratory data, our diagnosis was acute pneumonia and pleural empyema due to $M$. intracellulare. The treatment was changed to clarithromycin $(1,000$ $\mathrm{mg} /$ day), rifampicin $(600 \mathrm{mg} /$ day $)$, ethambutol $(1,200 \mathrm{mg} /$ day), and streptomycin (1 g intramuscular injection three times per week). A gradual improvement in the clinical features was observed with ongoing treatment. The patient was discharged with maintenance of above medications.

Four weeks later, numerous mycobacterial colonies were cultured from multiple specimens including six sputum samples and two pleural fluid collections on Ogawa's egg medium. All of these colonies were subsequently identified as $M$. intracellulare. The identification was accomplished via a PCR-restriction fragment length polymorphism method, based on the $r p o B$ gene (2). Presently, he is doing well on his four-drug regimen after 9 months of therapy (Fig. 2).

\section{Discussion}

This is a very rare case of acute pneumonia accompanied by pleural empyema due to $M$. intracellulare. NTM are ubiquitous organisms. They have been increasingly recognized as important causes of chronic pulmonary infection in immunocompetent individuals (1). Among the NTM, the Mycobacterium avium-intracellulare complex (MAC), consisting of two species, M. avium and M. intracellulare, constitutes the most commonly encountered pathogen of NTM pulmonary disease in a host of countries, including Korea $(1,3-7)$.

Although the clinical and radiological features of NTM infection resemble those of tuberculosis, NTM infection is rarely accompanied by pleural involvement. A study by Christensen et al examined the initial radiographic appearance of 100 cases with MAC pulmonary disease. Only 6\% had a defined edge of pleural thickening or fluid blunting a lateral or posterior costophrenic sulcus (8). Lynch et al reported the CT features of 55 cases with MAC pulmonary disease. Only $7 \%$ had pleural calcification (9).

There have been a few case reports of pleural effusion caused by NTM such as MAC (10-15), M. kansasii (16-18), and M. scrofulaceum (19). In addition, two cases of chronic empyema due to MAC were reported and these cases were managed with surgical treatment including pleuropneumonectomy or thoracoplasty $(20,21)$. It is interesting that most cases were reported from Japan. However, there has been no case report of acute pneumonia and pleural empyema caused by MAC as in the present.

It is generally assumed that when NTM is isolated from a closed space, it is responsible for the pathological changes observed. However, NTM can be isolated from pleural effusion unrelated to NTM infection, such as an in congestive heart failure and metastatic cancers (22). In the present case, 

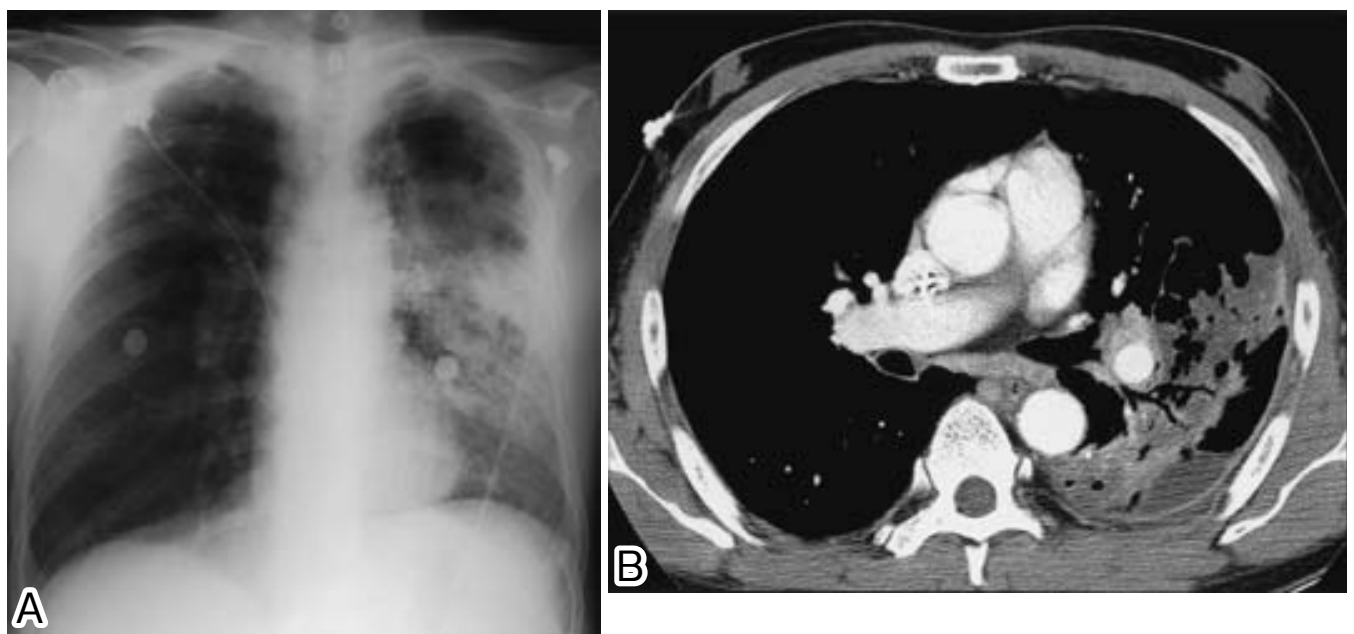

Figure 1. A 56-year-old man with Mycobacterium intracellulare pulmonary disease manifesting as acute pneumonia and empyema. A. The posteroanterior chest radiograph revealed a pneumonic consolidation in the left hemithorax. Multiple calcified nodular opacities in the both upper lobes and apical fibro-bullous changes in the left upper lobe are also seen. B. Contrast-enhanced chest computed tomography scan (5-mm collimation) revealed a parenchymal consolidation with airbronchogram in the left lower lobe. Note the hydropneumothorax and enhancing pleural thickening suggesting pleural empyema.

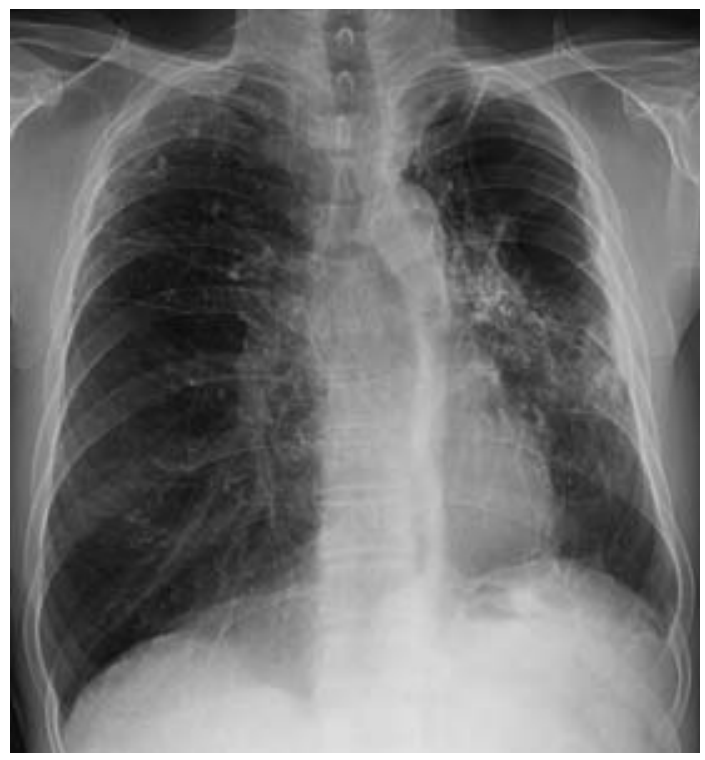

Figure 2. Posteroanterior chest radiograph after 9 months of antibiotic therapy including clarithromycin, rifampin, ethambutol, and streptomycin. The extent of pneumonic consolidation was remarkably decreased.

the patient did not have such an underlying disease. The clinical features of this case were typical of acute pneumonia associated with pleural empyema due to MAC. The patient had acute onset of fever, chills, purulent sputum and pleuritic chest pain. Radiographic findings revealed parenchymal consolidation and loculated hydropneumothorax. The aspirated pleural fluid was grossly purulent. We detected only MAC from a closed space, i.e. from the pleural space, and multiple sputum specimens, and did not detect any pathogenic microorganism. Therefore, we diagnosed the condition of this patient as pleural empyema caused by MAC.

The insidious nature of MAC pulmonary disease is well known, as symptoms may be present for months or years before the diagnosis is made (23). Most patients with MAC pulmonary disease experience chronic cough, usually productive of purulent sputum. Constitutional symptoms including fever, night sweats, weight loss, malaise, lethargy and fatigue are not common unless patients have extensive lung disease. The present patient was unusual considering this chronic indolent course of NTM pulmonary disease

The pathway from NTM infection to pleural empyema is unclear, but there are two theories about the process (21). The first theory is development of the empyema from the lung infection. The second theory entails the development of the empyema after a minor trauma. Computed tomography in the present case clearly demonstrated parenchymal consolidation and loculated hydropneumothorax. This finding suggests that pleural empyema in our case resulted from pulmonary parenchymal infection through a bronchopleural fistula. Bronchopleural fistula associated with tuberculosis usually follows a surgical procedure but can also occur spontaneously $(24,25)$. Our case showed that the development of spontaneous bronchopleural fistula due to pulmonary MAC infection could be possible. Although the exact reason for the development of pleural empyema is yet unknown, our case suggests that the fibro-bullous changes due to previous pulmonary tuberculosis could play a possible role in developing a bronchopleural fistula and subsequent pleural empyema due to MAC.

In summary, our case suggests that acute pneumonia and pleural empyema should be considered in patients with pulmonary diseases caused by MAC. Physicians should be 
aware of acute pneumonia and empyema caused by MAC in non-immunocompromised patients.

\section{References}

1. American Thoracic Society. Diagnosis and treatment of disease caused by nontuberculous mycobacteria. Am J Respir Crit Care Med 156: S1-S25, 1997.

2. Lee H, Park HJ, Cho SN, Bai GH, Kim SJ. Species identification of mycobacteria by PCR-restriction fragment length polymorphism of the rроB gene. J Clin Microbiol 38: 2966-2971, 2000.

3. Sakatani M. The non-tuberculous mycobacteriosis. Kekkaku 80 25-30, 2005 (in Japanese, Abstract in English).

4. Koh WJ, Kwon OJ, Lee KS. Nontuberculous mycobacterial pulmonary diseases in immunocompetent patients. Korean J Radiol 3: 145-157, 2002.

5. Jeon K, Koh WJ, Kwon OJ, et al. Recovery rate of NTM from AFB smear-positive sputum specimens at a medical centre in South Korea. Int J Tuberc Lung Dis 9: 1046-1051, 2005.

6. Koh WJ, Kwon OJ, Lee KS. Diagnosis and treatment of nontuberculous mycobacterial pulmonary disease: a Korean perspective. J Korean Med Sci 20: 913-925, 2005.

7. Koh WJ, Kwon OJ, Jeon K, et al. Clinical significance of nontuberculous mycobacteria isolated from respiratory specimens in Korea. Chest 129: 341-348, 2006

8. Christensen EE, Dietz GW, Ahn $\mathrm{CH}$, et al. Initial roentgenographic manifestations of pulmonary Mycobacterium tuberculosis, M. kansasii, and M. intracellularis infections. Chest 80: 132-136, 1981.

9. Lynch DA, Simone PM, Fox MA, Bucher BL, Heinig MJ. CT features of pulmonary Mycobacterium avium complex infection. J Comput Assist Tomogr 19: 353-360, 1995.

10. Okada Y, Ichinose Y, Yamaguchi K, Kanazawa M, Yamasawa F, Kawashiro T. Mycobacterium avium-intracellulare pleuritis with massive pleural effusion. Eur Respir J 8: 1428-1429, 1995.

11. Nagaia T, Akiyama M, Mita Y, Tomizawa T, Dobashi K, Mori M. Mycobacterium avium complex pleuritis accompanied by diabetes mellitus. Diabetes Res Clin Pract 48: 99-104, 2000.

12. Kawamoto H, Yamagata M, Nakashima H, et al. Development of a case of Mycobacterium avium complex disease from right pleural effusion. Nihon Kokyuki Gakkai Zasshi 38: 706-709, 2000 (in Japanese, Abstract in English).

13. Saitou M, Niitsuma K. A case of Mycobacterium avium lung infection with a pleural effusion. Kansenshogaku Zasshi 75: 504506, 2001 (in Japanese, Abstract in English).
14. Kobayashi K, Yano S, Kato K, Saito S, Tokushima T. A case of Mycobacterium avium pulmonary disease accompanied with pleural effusion. Kekkaku 77: 725-728, 2002 (in Japanese, Abstract in English).

15. Yanagihara K, Tomono K, Sawai T, et al. Mycobacterium avium complex pleuritis. Respiration 69: 547-549, 2002.

16. Igari $\mathrm{H}$, Kikuchi N. Nontuberculous mycobacterium pulmonary infection with pleural effusion caused by Mycobacterium kansasii. Kekkaku 68: 527-531, 1993 (in Japanese, Abstract in English).

17. Kamiya H, Toyota E, Kobayashi N, Kudo K. A case of pulmonary Mycobacterium kansasii infection complicated with pleural effusion. Kekkaku 79: 397-400, 2004 (in Japanese, Abstract in English).

18. Olafsson EJ, Naum CC, Sarosi GA, Mastronarde JG. Bilateral pleural effusions and right pneumothorax in a 25 -year-old man. Chest 126: 986-992, 2004.

19. Fusegawa H, Ookubo Y, Nishiumi M, Fujino T. A case of pulmonary Mycobacterium scrofulaceum infection presented as pleurisy. Kekkaku 80: 469-473, 2005 (in Japanese, Abstract in English).

20. Yamamoto H, Osako T, Aogauchi R, Yamamoto H, Hironaka S. Surgical treatment of Mycobacterium avium complex infection in a patient with chronic empyema. Nippon Geka Gakkai Zasshi 95: 123-125, 1994 (in Japanese, Abstract in English).

21. Kotani K, Hirose Y, Endo S, Yamamoto H, Makihara S. Surgical treatment of atypical Mycobacterium intracellulare infection with chronic empyema: A case report. J Thorac Cardiovasc Surg 130: 907-908, 2005.

22. Gribetz AR, Damsker B, Marchevsky A, Bottone EJ. Nontuberculous mycobacteria in pleural fluid. Assessment of clinical significance. Chest 87: 495-498, 1985.

23. Field SK, Fisher D, Cowie RL. Mycobacterium avium complex pulmonary disease in patients without HIV infection. Chest 126: 566-581, 2004.

24. Kim HY, Song KS, Goo JM, Lee JS, Lee KS, Lim TH. Thoracic sequelae and complications of tuberculosis. Radiographics 21: 839-858, 2001.

25. Lois M, Noppen M. Bronchopleural fistulas: an overview of the problem with special focus on endoscopic management. Chest 128: 3955-3965, 2005

(C) 2006 The Japanese Society of Internal Medicine http://www.naika.or.jp/imindex.html 